\title{
Seletividade de Herbicidas. III - AplicaÇÃo de Herbicidas em Pós-EMERgênCIA INICIAL E TARDIA dA CANA-DE-AçÚCAR NA ÉPOCA DA ESTIAGEM $^{1}$
}

\author{
Herbicide Selectivity. III - Herbicide Application at Initial and Late Postemergence of \\ Sugarcane in Dry Season \\ AZANIA, C.A.M. ${ }^{2}$, ROLIM, J.C. ${ }^{3}$, CASAGRANDE, A.A. ${ }^{4}$, LAVORENTI, N.A. ${ }^{5}$ e \\ AZANIA, A.A.P.M. ${ }^{6}$
}

\begin{abstract}
RESUMO - Este trabalho objetivou avaliar a seletividade dos herbicidas diuron+hexazinone, azafenidin+hexazinone, metribuzin e isoxaflutole aplicados em pós-emergência inicial e tardia das plantas de cana-de-açúcar, variedade RB835089, na época da estiagem, em soqueira de quarto corte, após colheita com queima prévia do canavial, em Araras, SP. O delineamento experimental foi o de blocos casualizados com testemunhas pareadas, com cinco tratamentos e quatro repetições, existindo uma testemunha capinada para cada tratamento químico dentro de cada bloco. Os tratamentos foram: diuron+hexazinone $\left(1.170+330 \mathrm{~g} \mathrm{ha}^{-1}\right)$, azafenidin+ hexazinone $\left(192,5+247,5 \mathrm{~g} \mathrm{ha}^{-1}\right)$, metribuzin $\left(1.920 \mathrm{~g} \mathrm{ha}^{-1}\right)$, isoxaflutole $\left(127,5 \mathrm{~g} \mathrm{ha}^{-1}\right) \mathrm{e}$ testemunha capinada. Concluiu-se que os herbicidas foram mais fitotóxicos quando aplicados na pós-emergência tardia. Em pós-emergência inicial, as plantas recuperaram-se totalmente dos efeitos fitotóxicos dos herbicidas. Na pós-emergência tardia, azafenidinthexazinone, isoxaflutole e diuronthexazinone prejudicaram o índice de fluorescência e a produtividade agrícola.
\end{abstract}

Palavras-chave: fitointoxicação, Saccharum spp.

\begin{abstract}
This research aimed to evaluate selectivity of azafenidin thexazinone, diuronthexazinone, metribuzin and isoxaflutole on sugarcane, applied early and during late postemergence in dry season. The study was conducted over 4-yr sugarcane ratoons (cv. RB 835089), in Araras, SP. The experimental design was a randomized block (four replications) with five treatments, plus one weed control to each chemical treatment. The treatments were: diuron+hexazinone $\left(1,170+330 \mathrm{~g} \mathrm{ha}^{-1}\right)$, azafenidin+hexazinone $(192,5+247,5 \mathrm{~g} \mathrm{ha}-1)$, metribuzin $\left(1,92 \mathrm{~L} \mathrm{ha}^{-1}\right)$ and isoxaflutole $\left(127,5 \mathrm{~g} \mathrm{ha}^{-1}\right)$ and weed control. Height, stand, chlorophyll, fluorescence index, production and technological characteristics were analyzed by the $F$ test and the "t" test, for average comparison. The herbicides caused more damage to the sugarcane in later postemergence. At initial postemergence, the herbicides did not cause any damage to the sugarcane. At later postemergence, azafenidin+hexazinone, isoxaflutole and diuronthexazinone damaged fluorescence index and sugarcane production.
\end{abstract}

Keywords: phytointoxication, Saccharum spp.

\footnotetext{
Recebido para publicação em 11.4.2005 e na forma revisada em 4.8.2006.

2 Pqc I Dr. do Centro de Cana IAC, Anel viário, Km 371, Caixa Postal 206, 14001-970 Ribeirão Preto-SP; ${ }^{3}$ Prof. Dr. do Centro de Ciências Agrárias, Universidade Federal de Santa Catarina - CCA/UFSCar. ${ }^{4}$ Prof. Dr. da Faculdade de Ciências Agrárias e Veterinárias, Universidade Estadual Paulista - FCAVJ/UNESP. ${ }^{5}$ Prof. Dr. do CCA/UFSCar; ${ }^{6}$ Doutoranda em Produção Vegetal na FCAVJ/UNESP.
} 


\section{INTRODUÇÃO}

No Estado de São Paulo, a cana-de-açúcar colhida durante o inverno (junho a agosto) apresenta lenta brotação e perfilhamento; entretanto, mesmo em menores densidades populacionais, as plantas daninhas podem prejudicar o desenvolvimento da cultura. Essas condições devem-se à pouca ocorrência de chuvas durante o inverno, característica dos climas Aw e Cwa de Köppen (Brasil, 1960), onde foi realizado o experimento e onde se encontra a maioria dos canaviais paulistas.

No entanto, muitos produtores, devido às questões de planejamentos e operações agrícolas, optam por utilizar o controle químico mesmo durante o período seco. Nesse caso, a seletividade dos herbicidas escolhidos deverá ser eficaz, para não prejudicar ainda mais o desenvolvimento das soqueiras.

Dentre os herbicidas a serem escolhidos pelos produtores, destaca-se a mistura pronta de azafenidin + hexazinone, que tem demonstrado seletividade para a cana-de-açúcar. Nesse sentido, Negrisoli (2002) e Pastre \& Rolim (2002) não observaram prejuízos à cultura ao utilizarem a mistura.

$\mathrm{O}$ isoxaflutole também se torna interessante, pois pode ser aplicado em períodos secos; segundo Lovell et al. (2000), ele se torna reativo somente na presença de chuva ou irrigação, controlando as plantas daninhas que emergirem. Esse herbicida, geralmente, intoxica a cana-de-açúcar nos primeiros meses após a aplicação, porém sem comprometer a produção, conforme pode ser observado em Costa \& Rozanski (2003). Os sintomas mais comuns são coloração branca (Pallett et al., 1998) e até queimaduras nas pontas das folhas.

O metribuzin também pode ser outra opção, pois apresenta, geralmente, menor toxicidade quando comparado a outros herbicidas, como pode ser verificando em Richard Jr. (1993, 1996), Millhollon (1993) e Miller et al. (1998).

Também pode ser empregada a mistura pronta de diuron + hexazinone, que, segundo Clement et al. (1979), Victoria Filho \& Camargo
(1980), Mello Filho et al. (1986) e Cruz \& Gurgell (1983), não prejudicou a produção nem os componentes da produção, como fibra, pol, brix e pureza da cana-de-açúcar.

Esses herbicidas podem ser aplicados em pré ou pós-emergência da cana-de-açúcar, porém é importante estudar seu comportamento no que se refere ao desenvolvimento da cana-de-açúcar quando aplicados no final do período de estiagem. Nesse caso, a seletividade do herbicida torna-se ainda mais importante, porque os sintomas de intoxicação podem ser mais severos.

Assim, considerando as condições em que há o período de menores temperaturas e ocorrência de chuvas, bem como o desenvolvimento lento das soqueiras de cana-deaçúcar e das plantas daninhas e os herbicidas descritos, esta pesquisa objetivou verificar a seletividade de azafenidin + hexazinone, isoxaflutole, metribuzin e diuron + hexazinone, aplicados em condição de pós-emergência inicial e tardia da cana-de-açúcar no final da época de estiagem, avaliada pelo delineamento em blocos casualizados com testemunhas pareadas, conforme metodologia proposta por Azania (2004).

\section{MATERIAL E MÉTODOS}

O experimento foi instalado no município de Araras, SP, no sítio Retiro, em solo de textura muito argilosa (61\% de argila, 24\% de areia e $15 \%$ de silte), previamente calcareado e adubado, de acordo com o resultado da análise de solo, para atender às necessidades nutricionais da cana-de-açúcar, soqueira de quarto corte, cv. RB835089.

A aplicação dos herbicidas foi realizada em dois momentos, constituindo duas áreas experimentais específicas. Na primeira, a aplicação dos herbicidas foi feita em pósemergência inicial da cana-de-açúcar, aos 20 dias após a colheita da terceira soca, quando as plantas apresentavam em média $9,58 \mathrm{~cm}$ de altura (do solo até a primeira folha completamente desenvolvida). Na segunda área experimental, a aplicação dos produtos foi realizada em pós-emergência tardia das plantas, aos 42 dias após a colheita, quando estas apresentavam altura média de $14,95 \mathrm{~cm}$. 
Os tratamentos, nas duas áreas, foram constituídos por diuron+hexazinone $(1.170+$ $\left.330 \mathrm{~g} \mathrm{ha}^{-1}\right)$, azafenidin+hexazinone $(192,5+$ $\left.247,5 \mathrm{~g} \mathrm{ha}^{-1}\right)$, metribuzin (1.920 $\mathrm{g} \mathrm{ha}^{-1}$ ), isoxaflutole $\left(127,5 \mathrm{~g} \mathrm{ha}^{-1}\right)$ e testemunha, sendo mantidas capinadas todas as parcelas durante todo o período experimental. Na aplicação utilizou-se equipamento costal pressurizado $\left(\mathrm{CO}_{2}\right)$, munido de barra com três bicos Teejet DG 110.02VS, espaçados de $0,50 \mathrm{~m}$, com pressão de 35 libras.pol $^{-2}$ e volume de calda de $250 \mathrm{~L} \mathrm{ha}^{-1}$.

O delineamento experimental utilizado em cada área experimental foi o de blocos casualizados, com cinco tratamentos e quatro repetições, salientando-se que existia uma testemunha para cada tratamento químico, dentro de cada bloco (delineamento em blocos casualizados com testemunhas pareadas), segundo Azania (2004). Portanto, em cada área experimental havia 16 parcelas tratadas com herbicidas e 16 parcelas testemunhas, colocadas pareadas a cada tratamento químico. As parcelas foram constituídas por cinco linhas de cana-de-açúcar com $10 \mathrm{~m}$ de comprimento, espaçadas de $1,30 \mathrm{~m}$. Foram consideradas como área útil as três linhas centrais de cada parcela (39 $\mathrm{m}^{2}$ por parcela).

A seletividade dos herbicidas às plantas de cana-de-açúcar foi avaliada visualmente aos 15, 30, 45 e 60 dias após aplicação (DAA), nas três linhas centrais de cada parcela, sendo atribuídas notas percentuais, utilizando-se uma escala variando de 0 a $100 \%$, sendo $0 \%$ nenhuma injúria e $100 \%$ morte total das plantas.

Na área útil de cada parcela também foram avaliados, aos 30, 45 e 60 DAA: a altura de dez plantas escolhidas ao acaso; o teor de clorofila total nas folhas (expresso em unidades relativas - UR), empregando-se clorofilômetro de campo (Spad 502 Minolta) no terço médio da folha +3 de seis plantas escolhidas ao acaso; e o índice de fluorescência ( $\mathrm{Fv} / \mathrm{Fm})$, empregando-se o fluorômetro portátil (PEA - "Plant Efficiency Analyser" Hansatech) no terço médio da folha +3 de seis plantas escolhidas ao acaso. Também foi determinado o número de colmos da área útil das parcelas, aos 90 e 180 DAA.

Por ocasião da colheita, com a soqueira apresentando 336 dias de ciclo, os valores percentuais de Brix\%caldo, Pol\%caldo,
Pureza\%caldo, Fibra\%cana, Açúcares Redutores\%caldo e Açúcar Teórico Recuperável\%caldo (ATR) foram detectados em laboratório, por amostragem de dez colmos retirados da linha central de cada parcela. O peso dos colmos das três linhas centrais de todas as parcelas foi avaliado, a fim de se determinar a produtividade agrícola ( $\left.\mathrm{tha}^{-1}\right)$.

Os resultados foram avaliados pela análise de variância com o teste $\mathrm{F}$, utilizando os dados coletados em todas as parcelas; para comparação das médias dos tratamentos foi utilizado o teste " $t$ " a $5 \%$ de probabilidade.

\section{RESULTADOS E DISCUSSÃO}

\section{Aplicação em pós-emergência inicial}

Nessa condição, todos os herbicidas causaram sintomas de intoxicação às plantas de cana-de-açúcar até os 45 DAA; aos 60 DAA não foram observados esses sintomas (Figura 1). Isoxaflutole e metribuzin foram mais tóxicos à cana-de-açúcar até os $15 \mathrm{DAA}$, porém aos 30 e 45 DAA apresentaram-se como os menos tóxicos. Na avaliação aos 15 DAT, as injúrias observadas em isoxaflutole foram um intenso amarelo com manchas brancas nas folhas da cultura, enquanto para metribuzin houve leve redução do porte das plantas.

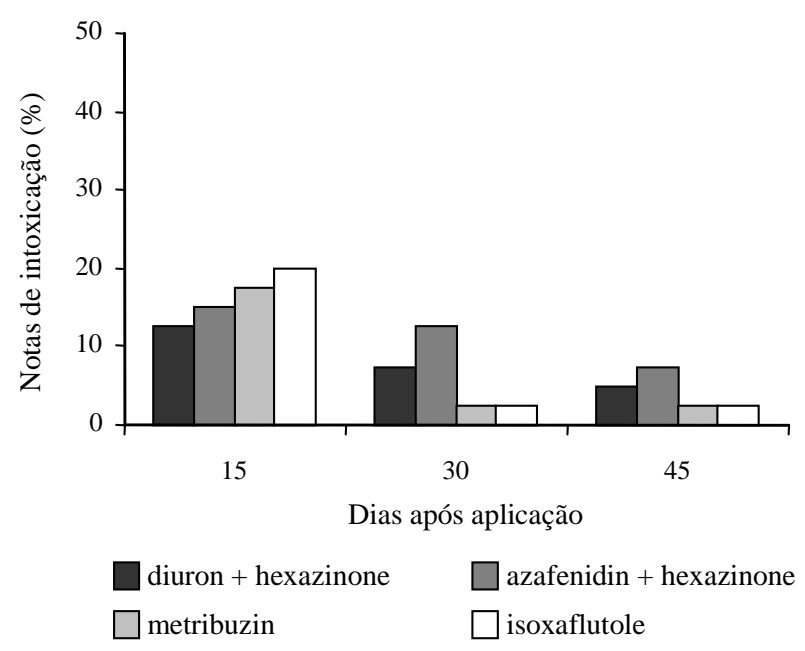

Figura 1 - Sintomas de intoxicação nas plantas de cana-deaçúcar aos 15, 30 e 45 DAA com diuron + hexazinone, azafenidin + hexazinone, metribuzin e isoxaflutole, em condição de pós-emergência inicial da soqueira (cultivar RB835089), durante a estação da seca. Araras, 2003. 
Por sua vez, azafenidin + hexazinone foi o terceiro mais tóxico à cana-de-açúcar aos 15 DAA, enquanto aos 30 e 45 DAA comportou-se como o mais tóxico, causando principalmente redução no porte das plantas. Também nesse período, diuron + hexazinone causou leve descoloração amarela nas folhas, que se apresentou mais intensa aos 15 DAA e, aos 30 e 45 DAA, apresentou-se mais recuperada.

Esses sintomas de intoxicação causados por todos os herbicidas possivelmente estão relacionados com a menor pluviosidade registrada desde o momento da aplicação dos herbicidas; a partir desse período até os 45 DAA foram registrados apenas $57,6 \mathrm{~mm}$ de chuva $(18,0+39,6 \mathrm{~mm})$, conforme a Tabela 1 .

Nessas condições de estiagem, os herbicidas podem ficar mais concentrados na camada superficial do solo, principalmente o isoxaflutole e o diuron + hexazinone, que, segundo Rodrigues \& Almeida (1998), adsorvem-se mais às partículas do solo. Nesse caso, a textura argilosa do solo da área experimental (61\% argila) pode ter colaborado ainda mais com a possivel adsorção e concentração desses herbicidas. Entretanto, o metribuzin (Rodrigues \& Almeida, 1998) e a mistura de azafenidin + hexazinone (ANVISA, 2003) são mais móveis no solo e possivelmente tenham ficado concentrados na camada superficial mesmo devido às poucas chuvas.

A altura aos 45 DAA foi prejudicada pela mistura pronta de diuron + hexazinone, metribuzin e isoxaflutole (Tabela 2), pois apresentaram médias menores que a da testemunha (contrastes 1,2 e 4), porém com total recuperação aos 60 DAA. Nesse período, apenas a mistura de azafenidin + hexazinone não

Tabela 1 - Precipitações pluviométricas e temperaturas ocorridas nas últimas décadas e durante o experimento. Araras, 2003

\begin{tabular}{|c|c|c|c|c|}
\hline \multirow{2}{*}{ Mês } & \multicolumn{2}{|c|}{ Precipitação (mm) } & \multicolumn{2}{|c|}{ Temperatura (qC) } \\
\hline & Média* & Experimento** & Média* & Experimento $* *$ \\
\hline Agosto $^{\frac{1}{1} / 02}$ & 33,5 & $110,2(18,0)^{\frac{3 /}{}}$ & 19,5 & 21,5 \\
\hline Setembro $^{2} / 02$ & 75,2 & 39,6 & 20,5 & 19,9 \\
\hline Outubro/02 & 123,0 & 73,0 & 22,1 & 25,6 \\
\hline
\end{tabular}

Fonte: CCA/UFSCar/Araras; * médias históricas mensais no período de 1.1 .1972 a 31.5.2003; ** dados mensais de precipitações e médias

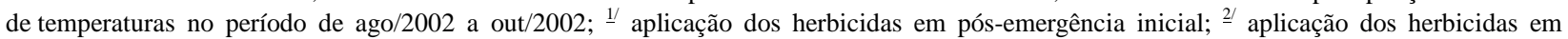

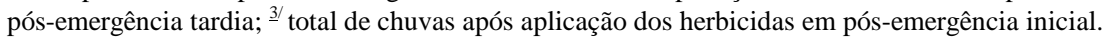

Tabela 2 - Altura, índice de fluorescência $(\mathrm{Fv} / \mathrm{Fm})$ e produtividade da cana-de-açúcar em resposta à aplicação de herbicidas em pós-emergência inicial e tardia. Araras, 2003

\begin{tabular}{|c|c|c|c|c|c|c|}
\hline \multicolumn{2}{|r|}{ Contrastes } & $\begin{array}{c}\text { Pós-emergência } \\
\text { inicial }\end{array}$ & \multicolumn{4}{|c|}{ Pós-emergência tardia } \\
\hline 1 & Testemunha vs. diuron+hexazinone & 95,04 vs. $87,39 * *$ & 40,78 vs $39,32 \mathrm{~ns}$ & 74,34 vs. $72,19 *$ & 76,69 vs. $74,37 * *$ & 152,32 vs. $129,87 * *$ \\
\hline 2 & Testemunha vs. metribuzin & 23,76 vs. $20,68 * *$ & 40,78 vs $40,50 \mathrm{~ns}$ & 74,34 vs. 73,06 ns & 76,69 vs. $73,63 \mathrm{~ns}$ & 152,32 vs. $143,43 \mathrm{~ns}$ \\
\hline 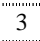 & Testemunha vs. azafenidin+hexazinone & 23,76 vs. $22,48 \mathrm{~ns}$ & 40,78 vs. $38,20 * *$ & 74,34 vs. $72,06 *$ & 76,69 vs. $75,00 *$ & 152,32 vs. $135,06 *$ \\
\hline 4 & Testemunha vs. isoxaflutole & 23,76 vs. $21,45 *$ & 40,78 vs. $37,93 * *$ & 74,34 vs. $70,50 * *$ & 76,69 vs. $74,88^{*}$ & 152,32 vs. $133,33 * *$ \\
\hline 5 & diuron+hexazinone vs. metribuzin & 23,76 vs. $22,78 \mathrm{~ns}$ & 39,32 vs. $40,50 \mathrm{~ns}$ & 72,19 vs. $73,06 \mathrm{~ns}$ & 74,37 vs. $73,63 *$ & 129,87 vs. $143,43 \mathrm{~ns}$ \\
\hline 6 & diuron+hexazinone vs. azafenidin+hexazinone & 20,68 vs. $22,48 \mathrm{~ns}$ & 39,32 vs. $38,20 \mathrm{~ns}$ & 72,19 vs. $72,06 \mathrm{~ns}$ & 74,37 vs. $75,00 \mathrm{~ns}$ & 129,87 vs. $135,06 \mathrm{~ns}$ \\
\hline 7 & diuron+hexazinone vs. isoxaflutole & 20,68 vs. $21,45 \mathrm{~ns}$ & 39,32 vs. $37,93 \mathrm{~ns}$ & 72,18 vs. $70,50 \mathrm{~ns}$ & 74,37 vs. $74,88 \mathrm{~ns}$ & 129,87 vs. $133,33 \mathrm{~ns}$ \\
\hline 8 & azafenidin+hexazinone vs. metribuzin & 20,68 vs. $22,78 \mathrm{~ns}$ & 38,20 vs. $40,50 *$ & 72,06 vs. $73,06 \mathrm{~ns}$ & 75,00 vs. $73,63 \mathrm{~ns}$ & 135,06 vs. $143,43 *$ \\
\hline - & azafenidin+hexazinone vs. isoxaflutole & 21,45 vs. $22,48 \mathrm{~ns}$ & 38,20 vs. $37,93 \mathrm{~ns}$ & 72,06 vs. $70,50 \mathrm{~ns}$ & 75,00 vs. $74,88 \mathrm{~ns}$ & 135,06 vs. $133,33 \mathrm{~ns}$ \\
\hline 10 & metribuzin vs. isoxaflutole & 21,45 vs. $22,78 \mathrm{~ns}$ & 40,50 vs. $37,93 *$ & 73,06 vs. $70,50 *$ & 73,63 vs. $74,88 \mathrm{~ns}$ & 143,43 vs. $133,33 \mathrm{~ns}$ \\
\hline & F tratamento & $2,81^{*}$ & $4,35 * *$ & $5,72 * *$ & $4,35 * *$ & ns \\
\hline & $\mathrm{CV}(\%)$ & 8,33 & 3,91 & 2,20 & 3,91 & 8,03 \\
\hline
\end{tabular}

Obs.: DAA (dias após aplicação); * significativo a 5\% de probabilidade pelo teste "t"; ** significativo a 1\% de probabilidade pelo teste "t"; ns não-significativo; $\mathrm{CV}=$ coeficiente de variação. 
prejudicou a altura, sendo observados alguns resultados semelhantes nos trabalhos de Negrisoli (2002) e Pastre \& Rolim (2002), quando verificaram que azafenidin + hexazinone não comprometeu a altura das plantas de canade-açúcar, a partir dos 45 DAA.

\section{Aplicação em pós-emergência tardia}

No experimento em pós-emergência tardia, observa-se pela Figura 2 que, no período de 15 a 45 DAA, os sintomas de intoxicação nas plantas foram mais severos, quando comparados com o experimento em pós-emergência inicial (Figura 1). Em ambas as situações, não foram observados esses sintomas aos 60 DAA.

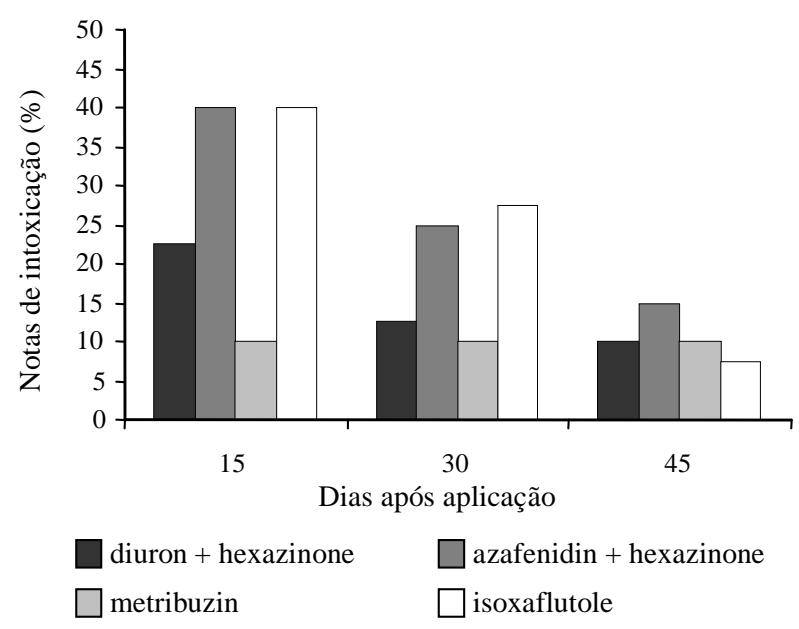

Figura 2 - Sintomas de intoxicação nas plantas de cana-deaçúcar aos 15, 30 e 45 DAA com diuron + hexazinone, azafenidin + hexazinone, metribuzin e isoxaflutole, em condição de pós-emergência tardia da soqueira (cultivar RB835089), durante a estação da seca. Araras, 2003.

Na Figura 2 observa-se que, entre os herbicidas aplicados, a mistura pronta de azafenidin + hexazinone foi a mais tóxica, causando manchas escuras nas folhas e plantas menos desenvolvidas. Esses sintomas podem estar relacionados com a maior quantidade de chuva (Tabela 1) e com a maior movimentação do herbicida pelo solo (ANVISA, 2003), que, talvez, tenham possibilitado seu maior contato com as raízes da cana e, conseqüentemente, maior absorção e os sintomas de intoxicação mais severos.
Outro herbicida também tóxico à cultura foi o isoxaflutole, que deixou as folhas amarelas (Figura 2). Esse sintoma pode estar relacionado com sua adsorção pelas partículas do solo (Rodrigues \& Almeida, 1998), possivelmente na mesma profundidade em que se encontravam as raízes da cana, acarretando maior absorção e sintomas de intoxicação mais severos. Entretanto, aos 45 DAA, o isoxaflutole apresentou-se como o herbicida menos tóxico, indicando rápida seletividade à cana-deaçúcar.

Quanto ao herbicida diuron + hexazinone, pode-se observar que os sintomas de intoxicação foram mais pronunciados que aqueles de metribuzin até os 30 DAA, ao passo que aos 45 DAA ambos apresentaram 10\% de injúrias na parte aérea das plantas (Figura 2). Nesse caso, o resultado da mistura de diuron + hexazinone comportou-se como em Cruz \& Gurgell (1983), ao constatarem que diuron + hexazinone foi mais tóxico que metribuzin até os 50 DAA. Todavia, quanto ao comportamento de metribuzin, Richard Jr. (1996, 1998) também verificou menores injúrias em canade-açúcar.

A altura aos 60 DAA também foi influenciada pelos herbicidas azafenidin + hexazinone e isoxaflutole (contrastes 3 e 4), conforme se pode verificar pela Tabela 2. Nessa época, ao se comparar a média de altura entre os tratamentos herbicidas, pode-se verificar que azafenidin + hexazinone e isoxaflutole apresentaram as menores alturas, diferindo de metribuzin (contrastes 8 e 10). Essas menores alturas para os tratamentos com azafenidin + hexazinone e isoxaflutole podem ser reflexo dos elevados sintomas de intoxicação observados até os 30 DAA (Figura 2).

A razão de fluorescência $(\mathrm{Fv} / \mathrm{Fm})$ foi prejudicada por diuron + hexazinone, azafenidin + hexazinone e isoxaflutole aos 45 e 60 DAA. Esses mesmos herbicidas também causaram sintomas de intoxicação elevados até os 30 DAA (Figura 2) e, com exceção de diuron + hexazinone, menor altura aos 60 DAA (Tabela 2). Nesse contexto, Cechin (1996) comentou que a razão $\mathrm{Fv} / \mathrm{Fm}$ para a maioria das culturas é de 0,8 (80\%); considerando que a cana apresentou valores entre 72 e $77 \%$ (Tabela 2), pode-se considerar que os tratamentos não foram muito prejudicados. 
Os tratamentos que influenciaram a produção final foram diuron + hexazinone e azafenidin + hexazinone, além do herbicida isoxaflutole (contrastes 1, 3 e 4), como se pode observar na Tabela 2. As diferenças ocorridas entre esses tratamentos e a testemunha foram de $22,45,17,25$ e 18,99 $\mathrm{t} \mathrm{ha}^{-1}$, para os tratamentos de diuron + hexazinone, azafenidin + hexazinone e isoxaflutole, respectivamente.

Em relação à quantidade de açúcar, ao se considerar ATR de 158, 16 kg para os tratamentos testemunhas, essas perdas correspondem

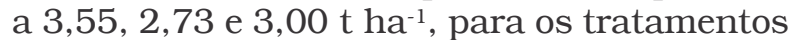
com diuron + hexazinone, azafenidin + hexazinone e isoxaflutole, respectivamente.

Esses resultados contrariam aqueles de Victoria Filho \& Camargo (1980) ao constatarem que diuron + hexazinone não prejudicou a produção, mas concordam com os desses autores quando se observa que essa mistura pronta não prejudicou as características tecnológicas. Essas contradições possivelmente devem ser devido às condições climáticas de cada experimento, além da maior precisão experimental do método estatístico. Entretanto, o comportamento de metribuzin foi semelhante àquele observado por Richard Jr. (1996, 1998), ao verificar que a produção final não foi prejudicada.

Assim, para a aplicação dos herbicidas em pós-emergência tardia da cana-de-açúcar, podem-se observar sintomas de intoxicação acentuados (Figura 2) e prejuízos para altura aos 60 DAA, indice de fluorescência aos 45 e 60 DAA e produção final. Esse resultados, possivelmente, podem ser atribuídos ao maior desenvolvimento das plantas no momento da aplicação dos herbicidas e ao aumento da ocorrência de chuva, logo após a aplicação dos herbicidas, aumentando a absorção destes e causando maiores prejuízos na parte aérea das plantas.

\section{LITERATURA CITADA}

AGÊNCIA NACIONAL DE VIGILÂNCIA SANITÁRIA ANVISA. Sistema de informação sobre agrotóxicos. Disponível em: <http://www4.anvisa.gov.br>. Acesso em: 4 out. 2003.

AZANIA, C. A. M. Comparação de métodos para determinar a seletividade de herbicidas na cultura da cana-de-açúcar. 2004. 116 f. Tese (Doutorado em Produção Vegetal) - Universidade Estadual Paulista, Jaboticabal, 2004.
BRASIL. Ministério da Agricultura. Serviço Nacional de Pesquisas Agronômicas. Comissão de Solos. Levantamento de Reconhecimento dos Solos do Estado de São Paulo. B. Serv. Nac. Pesq. Agron., v. 1, n. 12, 1960. $312 \mathrm{p}$.

CECHIN, I. Uso de sistemas portáteis de fluorescência na avaliação do estresse. In: CONGRESSO DA SOCIEDADE BOTÂNICA DE SÃO PAULO, 20., 1996, São Carlos. Anais... São Carlos: Universidade Federal de São Carlos, 1996. p. 1-28.

CLEMENT, A. A.; LAMMEL, J. S.; BARBOSA, J. C. Controle de plantas daninhas em cana-de-açúcar (Saccharum officinarum) com hexazinone e suas misturas com diuron em pré-emergência. Planta Daninha, v. 2, p. 85-88, 1979.

COSTA, E. A. D.; ROZANSKI, A. Eficácia da aplicação seqüencial de isoxaflutole associado com ametryn ou diuron no controle de plantas daninhas em cana-de-açúcar. B. Inf. SBCPD, v. 9, n. 1, p. 14-20, 2003.

CRUZ, L. S. P.; GURGELL, M. N. A. Efeito de hexazinone e diuron, e suas misturas, no controle de capim-colchão (Digitaria sanguinalis) em cana-de-açúcar (Saccharum spp.). Planta Daninha, v. 1, p. 15-20, 1983.

LOVELL, S. T. et al. Hidrolysis and soil adsorption of the labile herbicide isoxaflutole. Environ. Sci. Technol., v. 34, p. 3186-3190, 2000.

MELLO FILHO, A. T. et al. Estudo comparativo de herbicidas de pós-emergência para o controle de plantas daninhas na cana-de-açúcar. STAB: Açúcar, Álcool Subprod., v. 5, n. 1, p. 32-36, 1986.

MILLER, D. K.; RICHARD JR, E. P.; GRIFFIN, J. L. Sugarcane (Saccharum spp. Hybrids) response to simulated fallow field residues of metribuzin and sulfometuron. Weed Technol., v. 12, p. 454-457, 1998.

MILLHOLLON, R. W. Preemergence control of ichgrass (Rottboellia cochinchinensis) and johnsongrass (Sorghum halepense) in sugarcane (Saccharum spp. Hybrids) with pendimenthalin and prodiamine. Weed Sci., v. 41, p. 621-626, 1993.

NEGRISOLI, E. Seletividade de herbicidas aplicados em pré-emergência e associados a nematicidas, à cultura da cana-de-açúcar, cultivar RB855113. 2002. $48 \mathrm{f}$. Dissertação (Mestrado em Agricultura) - Universidade Estadual Paulista, Botucatu, 2002.

PALLETT, K. E. et al. The mode of action of isoxaflutoleI. Physiological effects, metabolism and selectivity. Pest. Biochem. Physiol., v. 62, p. 113-124, 1998. 
PASTRE, W.; ROLIM, J. C. Tolerância de variedades de cana-de-açúcar ao Êvolus - cana planta de 12 meses, em solos arenoso e argiloso. CONGRESSO BRASILEIRO DA CIÊNCIA DAS PLANTAS DANINHAS, 23., 2002,

Gramado. Resumos... Gramado: SBCPD, 2002. p. 490.

RICHARD JR, E. P. Preemergence herbicide effects on bermudagrass (Cynodon dactylon) interference in sugarcane (Saccharum spp. Hybrids). Weed Technol., v. 7, p. 578-584, 1993.

RICHARD JR., E. P. Sugarcane (Saccharum spp. Hybrids) tolerance to clomazone. Weed Technol., v. 10, p. 90-94, 1996.
RICHARD JR., E. P. Control of perennated bermudagrass (Cynodon dactylon) and johnsongrass (Sorghum halepense) in sugarcane (Saccharum spp. Hybrids). Weed Technol., v. 12, p. 128-133, 1998.

RODRIGUES, B. N.; ALMEIDA, F. S. Guia de herbicidas. 4.ed. Londrina: 1998. p. 228-232, 361-365, 389-395.

VICTORIA FILHO, R.; CAMARGO, P. N. Efeito de herbicidas nos teores de macronutrientes e nas características tecnológicas da cana-de-açúcar (Saccharum spp.). I - mistura de herbicidas em pós-emergência. Planta Daninha, v. 2, p. 96-107, 1980. 